OPEN ACCESS

Edited by:

David William Waite,

The University of Queensland,

Australia

Reviewed by:

Gerardo Zuniga

Instituto Politécnico Nacional, Mexico

Zhanghong Shi,

Fujian Agriculture and Forestry

University, China

*Correspondence:

Min Lu

lumin@ioz.ac.cn

tThese authors have contributed equally to this work as co-first authors.

Specialty section:

This article was submitted to

Microbial Symbioses,

a section of the journal

Frontiers in Microbiology

Received: 29 March 2018

Accepted: 23 May 2018

Published: 14 June 2018

Citation:

Xu D, Xu L, Zhou F, Wang B, Wang $S$,

Lu M and Sun J (2018) Gut Bacterial Communities of Dendroctonus valens and Monoterpenes and Carbohydrates of Pinus tabuliformis at Different Attack

Densities to Host Pines.

Front. Microbiol. 9:1251.

doi: 10.3389/fmicb.2018.01251

\section{Gut Bacterial Communities of Dendroctonus valens and Monoterpenes and Carbohydrates of Pinus tabuliformis at Different Attack Densities to Host Pines}

\author{
Dandan $\mathrm{Xu}^{1+}$, Letian $\mathrm{Xu}^{1,2+}$, Fangyuan Zhou ${ }^{1,3+}$, Bo Wang ${ }^{4}$, Shanshan Wang ${ }^{1,5}$, Min $\mathrm{Lu}^{1 *}$ \\ and Jianghua Sun ${ }^{1}$
}

\begin{abstract}
'State Key Laboratory of Integrated Management of Pest Insects and Rodents, Institute of Zoology, Chinese Academy of Sciences, Beijing, China, ${ }^{2}$ Hubei Collaborative Innovation Center for Green Transformation of Bio-Resources, College of Life Science, Hubei University, Wuhan, China, ${ }^{3}$ Shandong Provincial Key Laboratory of Applied Microbiology, Ecology Institute, Qilu University of Technology (Shandong Academy of Sciences), Jinan, China, ${ }^{4}$ Key Laboratory of Tropical Forest Ecology, Xishuangbanna Tropical Botanical Garden, Chinese Academy of Sciences, Menglun, China, ${ }^{5}$ Institute of Health Sciences, Anhui University, Hefei, China
\end{abstract}

Insects harbor a community of gut bacteria, ranging from pathogenic to obligate mutualistic organisms. Both biotic and abiotic factors can influence species composition and structure of the insect gut bacterial communities. Dendroctonus valens is a destructive forest pest in China. To overcome host pine defenses, beetles mass-attack the pine to a threshold density that can exhaust pine defenses. The intensity of pine chemical defenses and carbohydrate concentrations of pines can be influenced by beetle attack, both of which are known factors that modify beetle's gut microbiota. However, little is known to what extent variation exists in the beetle's gut communities, and host monoterpenes and carbohydrates at different attack densities. In this study, the gut bacterial microbiota of $D$. valens at low and high attack densities were analyzed, and monoterpenes and carbohydrates in host pine phloem were assayed in parallel. The results showed that no significant changes of gut bacterial communities of the beetles and concentrations of D-glucose, D-pinitol, and D-fructose in pine phloem were found between low and high attack densities. The concentrations of $\alpha$-pinene, $\beta$-pinene, limonene at high attack densities were significantly higher than those at low attack densities. Our results suggested that different attack densities of $D$. valens influence monoterpenes concentration of host pines' phloem but have no significant impact on gut bacterial community structures of $D$. valens and carbohydrate concentration of host trees' phloem in early attack phase. Similar gut bacterial community structures of $D$. valens between low and high attack densities might be due to the quick adaptation of gut microbiota to high monoterpenes concentrations.

Keywords: Dendroctonus valens, gut microbiota, monoterpenes, carbohydrates, attack density 


\section{INTRODUCTION}

The intestinal tract of insects is colonized by a dense microbiota composed of diverse communities ranging from pathogenic to obligate mutualistic organisms (Dillon and Dillon, 2004; Engel and Moran, 2013). The gut microbes of insects have been shown to confer advantages to the host in terms of nutrient provision (Visotto et al., 2009; Muhammad et al., 2017), suppression of pathogens and parasites (Koch and Schmid-Hempel, 2011; Engel and Moran, 2013), detoxification of xenobiotics (Genta et al., 2006; Kikuchi et al., 2012; Xu et al., 2016a), pheromone production (Dillon et al., 2002; Cao et al., 2018), regulation of immune response (Rodrigues et al., 2010; Blumberg et al., 2013), and influencing insect behavior (Sharon et al., 2010). Besides insect taxonomic position and physicochemical environment of the insect gut, host's diet and plant defensive chemicals are well known factors that are capable of significantly influencing herbivore insects gut bacterial communities (Colman et al., 2012; Engel and Moran, 2013; Mason et al., 2015).

Bark beetles (Coleoptera: Curculionidae: Scolytinae), a group of subcortical insects that feed as larvae and adults in the phloem of trees and woody shrubs (Coulson, 1979), have caused widespread coniferous tree mortality and severe economic losses around the globe (Paine et al., 1997; Gitau et al., 2013; Sun et al., 2013). Coniferous trees can produce defensive chemicals, such as monoterpenes, in defense against bark beetle attack (Smith, 1963; Byers, 1981; Phillips and Croteau, 1999; Seybold et al., 2006). Previous studies suggested that both the intensity of host chemical defenses and carbohydrate concentration are influenced by beetles' attack (Miller et al., 1986; Leufvén and Birgersson, 1987). Gut bacterial communities of bark beetle were shown to be less diverse than that of other insects (Vasanthakumar et al., 2006; Durand et al., 2015; BrionesRoblero et al., 2017; Hernández-García et al., 2017), which may be linked to hosts' diet, plant defensive chemicals, and gut's environment that exert a selective pressure on the diversity of bacterial species (Colman et al., 2012; Engel and Moran, 2013; Mason et al., 2015; Hernández-García et al., 2017). Furthermore, gut bacteria of bark beetles were reported to possess many beneficial ecological functions including degradation of defensive chemicals and nutrition provision (Boone et al., 2013; MoralesJiménez et al., 2013; García-Fraile, 2018; Howe et al., 2018). These previous studies described a complex and also elusive interaction between gut microbiota of beetles and host pines. Although gut bacteria communities of bark beetles at different life stages have been investigated (Vasanthakumar et al., 2006; Briones-Roblero et al., 2017; Durand et al., unpublished), little is known about how gut bacterial communities of beetles vary in parallel with carbohydrates and defensive chemicals concentration changes of host pines at different attack densities.

Dendroctonus valens LeConte (Coleoptera, Curculionidae, Scolytinae) is a destructive pine-killing invasive pest in China, which was introduced in the early 1980s from North America and has killed more than ten million Pinus tabuliformis Carrière trees thus far (Yan et al., 2005; Sun et al., 2013). Pioneer beetles arrive at susceptible pine trees and attract conspecifics to the host tree (Wood, 1982; Sullivan, 2016). Beyond a critical attack density threshold, host tree defenses are exhausted (e.g., monoterpenes defense), resulting in beetle establishment (Raffa and Berryman, 1983; Guérard et al., 2000). Beetles with low attack density are unable to colonize the trees and are typically killed by host pine defenses (Hedden and Pitman, 1978; Gao et al., 2005). Gut bacteria of $D$. valens have been shown to degrade host defensive monoterpenes in vitro and affect carbohydrate allocation in the consumed host tissue to benefit larval development (Xu et al., 2016a; Zhou et al., 2016), both of which influence bacterial community structure. A stable gut bacterial community is important for community function (Xu et al., 2016c), however, exactly how the bacterial communities change in response to fluctuations in host carbohydrates and defensive chemical concentrations during attack at different densities of beetles remains to be determined.

The purpose of this study was to evaluate the effect of low and high attack densities on gut bacterial community structure of D. valens, defensive chemicals and carbohydrate concentrations of host pines. We also discussed the connection between the change in bacterial community structure and defensive monoterpenes and carbohydrate variation of host pines, which may reveal how gut bacterial communities facilitate successful attack by $D$. valens.

\section{MATERIALS AND METHODS}

\section{Insects and Samples}

Adult beetles were collected from the Lindgren funnel traps baited with kairomone lure $[(+)-\alpha$-pinene: $(-)-\beta$-pinene: $(+)-$ 3 -carene $=1: 1: 1] \quad(99 \%, 98 \%, 97 \%$ respectively. Sigma-Aldrich, China) in the Tunlanchuan Forestry Station (N $37^{\circ} 48^{\prime}$, E $111^{\circ}$ $44^{\prime}$, average elevation 1,400 m), west of Gujiao City, Shanxi province in July 2015. Sexes of bark beetles were distinguished based on the stridulation of males (Lyon, 1958). Uninfested P. tabuliformis trees were cut into $50 \mathrm{~cm}$ lengths (diameter $\geq 30 \mathrm{~cm}$ ), and both ends of the bolts were immediately dipped into melted paraffin to delay desiccation. Three evenly spaced holes ( $80 \mathrm{~mm}$ in diameter) were drilled into each bolt, and a pair of adult beetles was introduced into each of the predrilled holes. The holes were secured with wire mesh (mesh size, $2.0 \mathrm{~mm}$ ). The bolts were checked every $24 \mathrm{~h}$ until each pair of beetles entered the bark. If they failed to enter, a new pair of beetles was introduced. The bolts were placed vertically in plastic boxes $(40 \mathrm{~cm}$ in diameter, $50 \mathrm{~cm}$ height). The lids of plastic boxes were open to keep air flowing before beetles emerged from the bolts and were closed to collect beetles when beetles emerged from bolts. The containers were stored at room temperature/humidity throughout the rearing period.

In June 2016, we randomly collected 480 adult beetles (240 females and 240 males) that emerged from these bolts and randomly chose 8 healthy $P$. tabuliformis pines $(\leq 100 \mathrm{~m}$ apart, the average diameter at breast height is $38.3 \pm 1.2 \mathrm{~cm})$ in the Tunlanchuan Forestry Station (N $37^{\circ} 48^{\prime}$, E $111^{\circ} 44^{\prime}$, average elevation 1,400 m), west of Gujiao City, Shanxi province. Four pines were set as high density group, and one hundred adult beetles (50 females and 50 males) were introduced into 
the main stem of each pine at $0.2-0.7 \mathrm{~m}$ height using the methods described above. The other four were set as low density group, and 20 adult beetles (10 females and 10 males) were introduced into each tree. After $72 \mathrm{~h}$, almost all beetles have bored in the phloem with the whole body under the surface and constructed 4-6 cm length galleries, which is considered as successful colonization (Birgersson et al., 1984; Zhang et al., 2000). We then dissected the phloem tissue to collect adult beetles and excise phloem tissues around inoculation point ( $5 \mathrm{~mm}$ around the inoculation points) in each tree. Complete guts were stretched out by cutting the head and separating the abdomen from the thorax of beetles, and gut samples that missed foregut, midgut or hindgut were discard. Each gut sample $(n=9)$ was put into a $2 \mathrm{~mL}$ Eppendorf tube, stored at $-80^{\circ} \mathrm{C}$ for DNA extraction. These phloem tissues $(n=12)$ were immediately frozen in liquid nitrogen for monoterpene ( $\alpha$-pinene, $\beta$-pinene, limonene) and carbohydrate (D-glucose, $\mathrm{D}$-pinitol, and D-fructose) quantification.

\section{DNA Extraction, PCR, Illumina MiSeq, and Sequence Processing}

DNA extraction from each adult beetle gut sample from two groups (high attack density group and low attack density group) was carried out using the TIANamp Bacteria DNA kit (TianGen, China) according to the manufacturer's instructions. The V3-V4 region of $16 \mathrm{~S}$ rRNA gene was amplified from the bacterial DNA by polymerase china reaction (PCR) using $16 \mathrm{~S}$ rRNA primers 341F ( $5^{\prime}$-CCTAYGGGRBGCASCAG-3') and 806R (5' - GGACTACHVGGGTWTCTAAT-3') (Xu et al., 2016c). The PCR reaction mixture contained $10 \mathrm{ng}$ of DNA, $1 \mu \mathrm{L}$ of $10 \mu \mathrm{M}$ of each primer, $2 \mu \mathrm{L}$ of $2.5 \mathrm{mM}$ dNTPs, $0.3 \mu \mathrm{L}$ Fastpfu polymerase (Transgene, China), and $4 \mu \mathrm{L} 5 \times$ Fastpfu buffer in a $20 \mu \mathrm{L}$ final volume. The PCR were carried out in an ABI GeneAmp ${ }^{\circledR}$ 9700 thermal cycler, cycling conditions were: $95^{\circ} \mathrm{C}$ for $10 \mathrm{~min}$; followed by 30 cycles of $95^{\circ} \mathrm{C}$ for $30 \mathrm{~s}, 55^{\circ} \mathrm{C}$ for $30 \mathrm{~s}$ and $72^{\circ} \mathrm{C}$ for $45 \mathrm{~s}$; followed by the final extension at $72^{\circ} \mathrm{C}$ for $10 \mathrm{~min}$. Each sample was amplified in three technical replicates $20 \mu \mathrm{L}$ PCR reaction and subsequently pooled together. The final PCR products were purified on $1.5 \%$ agarose gel by electrophoresis. Sequencing was performed on an Illumina platform (Illumina MiSeq PE250).

The sequencing data were preprocessed. Sequences were assigned to samples according to specific barcodes and removed barcodes and primers. Paired-end reads were assembled with FLASH $\left(\mathrm{V} 1.2 .7^{1}\right)$. High-quality data (clean reads) were acquired using the QIIME (Quantitative Insights Into Microbial Ecology) software packages $\left(\mathrm{V} 1.9 .0^{2}\right)$ by filtering low-quality data with default parameters (Caporaso et al., 2010). Chimeric sequences were detected and removed using UCHIME Algorithm (Edgar et al., 2011). All effective reads from each sample were initially clustered into Operational Taxonomic Units (OTUs) of 97\% sequence similarity with a UPARSE algorithm (Edgar, 2013). The most abundant sequence in each OTU was selected as the

${ }^{1}$ http://ccb.jhu.edu/software/FLASH/

${ }^{2} \mathrm{http}: / /$ qiime.org/index.html representative OTU using Greengene database ${ }^{3}$ and annotated by the RDP classifier algorithm implemented in QIIME under a confidence threshold of $80 \%$ (DeSantis et al., 2006; Wang et al., 2007). The raw sequence reads were obtained and deposited in the NCBI Sequence Read Archive under accession number SRR5349096 (reference: BioProject PRJNA379332).

For MiSeq data analysis, rarefaction curves were estimated using the 'alpha_rarefaction.py' script in QIIME to test whether the sequencing efforts adequately represented the bacterial diversity within each sample. Two richness estimators (the abundance-based coverage estimator (ACE) and a nonparametric richness estimator based on distribution of singletons and doubletons (Chaol) and two diversity indices (Shannon and Simpson index) were calculated for the samples using the 'alpha_diversity.py' script in QIIME. The diversity indices of two groups and the relative abundances of different genera were compared using an independent $t$-test. Non-metric multidimensional scaling (NMDS) was used to visualize the sample groupings based on Bray-Curtis similarity. Composition differences were tested using ANOSIM with 10000 permutations using PAST software, version 3.05 (Hammer et al., 2001). The representative sequences of all OTUs were used to construct neighbor-joining trees with QIIME. The phylogenetic tree together with sample sequence abundance data was used for weighted Unifrac PCoA (principal coordinate analysis) which considers both relative abundance and different branch lengths in a tree, through the online Fast Unifrac program (Hamady et al., 2010). A Permutational Multivariate Analysis of Variance based on the weighted UniFrac distance (PERMANOVA, "PermanovaG" function in the "GUniFrac" package of R) was used to test for differences in community composition between two sample groups.

\section{Monoterpenes Concentration in the Phloem Tissue of Trees at Two Attack Densities}

The dissected phloem tissues were weighed and then ground under liquid nitrogen until a fine dry powder was obtained. Twelve phloem powder samples from each group (high attack density group, low attack density group) were extracted with hexane containing an internal standard (heptyl acetate) separately and then stored at $-20^{\circ} \mathrm{C}$ for the chemical analysis. The most three abundant monoterpenes ( $\alpha$-pinene, $\beta$-pinene, Limonene) in the phloem were assayed (Leufvén and Birgersson, 1987; Xu et al., 2014).

Extracts $(2 \mu \mathrm{L})$ were injected splitless into a gas chromatography-mass spectrometer (GC-MS: Agilent 6980N GC coupled 5973 mass selective detector) equipped with an HP5-MS capillary column $(0.25 \mathrm{~mm}$ internal diameter $\times 30 \mathrm{~m}$; Agilent Technologies, Inc., Palo Alto, CA, United States), and the column temperature was programmed from an initial temperature of $50^{\circ} \mathrm{C}$ for $1 \mathrm{~min}$, then increased by $5^{\circ} \mathrm{C} / \mathrm{min}$ to $100^{\circ} \mathrm{C}$, by $3^{\circ} \mathrm{C} / \mathrm{min}$ to $130^{\circ} \mathrm{C}$, and by $20^{\circ} \mathrm{C}$ to $320^{\circ} \mathrm{C}$ and held for $2 \mathrm{~min}$. Components of the extracts were identified by comparing retention times

${ }^{3}$ http://greengenes.secondgenome.com/ 
and mass spectra with authentic standards and those in the NIST02 library (Scientific Instrument Services, Inc., Ringoes, NJ, United States). Quantification was performed using an internal standard (heptyl acetate) that was added to each sample.

\section{Carbohydrates (D-glucose, D-pinitol, and D-fructose) Concentration in the Phloem Tissue of Trees at Two Attack Density Groups}

Twelve phloem powder samples from each group, high density group, low density group, were extracted by the method described by Lisec et al. (2006) with little modification. Two hundreds mg samples and $5 \mathrm{~mL} 100 \%$ methanol were put into a $10 \mathrm{~mL}$ centrifuge tube and shaken for $20 \mathrm{~min}$ at $70^{\circ} \mathrm{C}$ in a thermomixer at $950 \mathrm{rpm}$. After the samples were centrifuged for $10 \mathrm{~min}$ at $11000 \times g$ and $350 \mu \mathrm{L}$ supernatant with $80 \mu \mathrm{L} 0.2 \mathrm{mg} / \mathrm{mL}$ ribitol as an internal quantitative standard was transferred into $350 \mu \mathrm{L}$

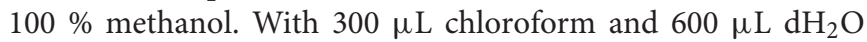
added, the samples were vortexed for $30 \mathrm{~s}$ and centrifuged at $2200 \times g$ for $20 \mathrm{~min}$, and $150 \mu \mathrm{L}$ of supernatant was transferred to a new $1.5 \mathrm{~mL}$ centrifuge tube. After the extracts were dried in a vacuum container, $40 \mu \mathrm{L}$ of methoxyamination reagent were added into the samples and the mixtures were shaken at $37^{\circ} \mathrm{C}$ for $3 \mathrm{~h}$. Then $70 \mu \mathrm{L}$ of MSTFA reagent were added into samples and the mixtures were shaken at $37^{\circ} \mathrm{C}$ for $30 \mathrm{~s}$. The supernatant was filtered with sodium sulfate anhydrous and kept in $2 \mathrm{~mL}$ vials (Agilent, United States) at $-20^{\circ} \mathrm{C}$ for chemical analysis.

Quantification analysis was carried on GC (Aglient 7890A) and FID (flame ionization detector). Chromatography conditions were as follows: injection volume $1 \mu \mathrm{L}$ without split, helium as carrier gas at $1 \mathrm{~mL} / \mathrm{min}$ constant flow mode, injector temperature $230^{\circ} \mathrm{C}$, HP-5 silica capillary column $(60 \mathrm{~m} \times 0.25 \mathrm{~mm} \times 0.25 \mu \mathrm{m})$. Oven temperature program was isothermal for $5 \mathrm{~min}$ at $70^{\circ} \mathrm{C}$, followed by a $5^{\circ} \mathrm{C}$ per min ramp to $310^{\circ} \mathrm{C}$, and holding at this temperature for $12 \mathrm{~min}$. Standard carbohydrates (D-glucose, D-pinitol, and D-fructose) were also tested by GC-FID to check the retention time, by which components of extracts were identified. Quantification was performed using an internal standard (ribitol) that was added to each sample.

Prior to analysis, we tested all variables for normality with the Kolmogorov-Smirnov test and homogeneity of group variances with Levene's test, and data were analyzed using independentsamples $t$-test. Differences between two groups were considered as significant when $P<0.05$. Data were analyzed using SPSS 12.0 (SPSS Inc., Chicago, IL, United States) and figures were drawn using Origin 8.5 (Origin Lab Corporation, Northampton, MA, United States).

\section{RESULTS}

\section{Illumina MiSeq Sequencing Data and $\alpha$-Diversity Analysis}

In the 18 representative gut samples, we obtained a total of 681138 sequences $(90.5 \%$ of the total trimmed 752331 ) and grouped into 1236 OTUs at 97\% similarity cut-off level. Rarefaction curves of the 18 gut samples almost reached equilibrium, which indicated that our Illumina MiSeq analysis covered the natural bacterial diversity well (Figure 1). Twentysix phyla were detected in the microbiota from 18 samples associated with D. valens, and of these 26 phyla, five main phyla (Proteobacteria, Firmicutes, Bacteroidetes, Actinobacteria, and Deinococcus-Thermus) representing more than $0.01 \%$ of total reads (Supplementary Table S1). At genus level, the sequences could be assigned to 141 genera (Supplementary Table S1). There were no significant differences between high density group and low density group in all five diversity indices (Table 1).

\section{$\beta$-Diversity Analysis}

An NMDS ordination analysis based on Bray-Curtis similarities across the samples suggested that the gut bacterial communities of $D$. valens in the low attack density group were similar to those in the high attack density group (Figure 2A; ANOSIM, $P=0.54)$. No separation was obtained in phylogeny-based weighted UniFrac principal coordinate analysis, which was confirmed by PERMANOVA (Figure 2B, $P=0.57$ ).

\section{OTU and Genus Abundance Analysis}

No difference in OTUs abundance between the two groups were found (Supplementary Table S1). At the genus level, the genera with an abundance of at least $0.01 \%$ of the total of number of reads were present in Supplementary Table S2. The relative

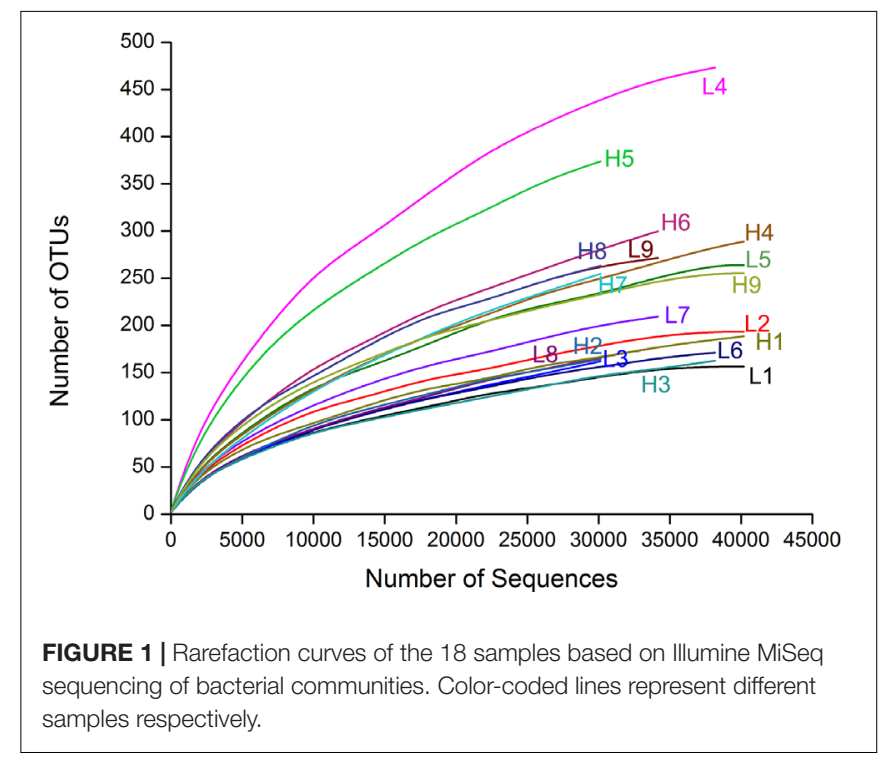

TABLE 1 | Comparison of diversity indices (Mean \pm SEM) of Dendroctonus valens gut bacterial community between the low and high density groups.

\begin{tabular}{lcc}
\hline Index & Low density & High density \\
\hline Number of OTUs & $273.67 \pm 55.26$ & $248.78 \pm 21.43$ \\
ACE & $417.26 \pm 63.77$ & $412.43 \pm 35.18$ \\
Chao1 & $387.52 \pm 57.67$ & $373.15 \pm 30.74$ \\
Shannon diversity $(H)$ & $1.71 \pm 0.17$ & $1.76 \pm 0.17$ \\
Simpson diversity & $0.49 \pm 0.04$ & $0.51 \pm 0.04$
\end{tabular}



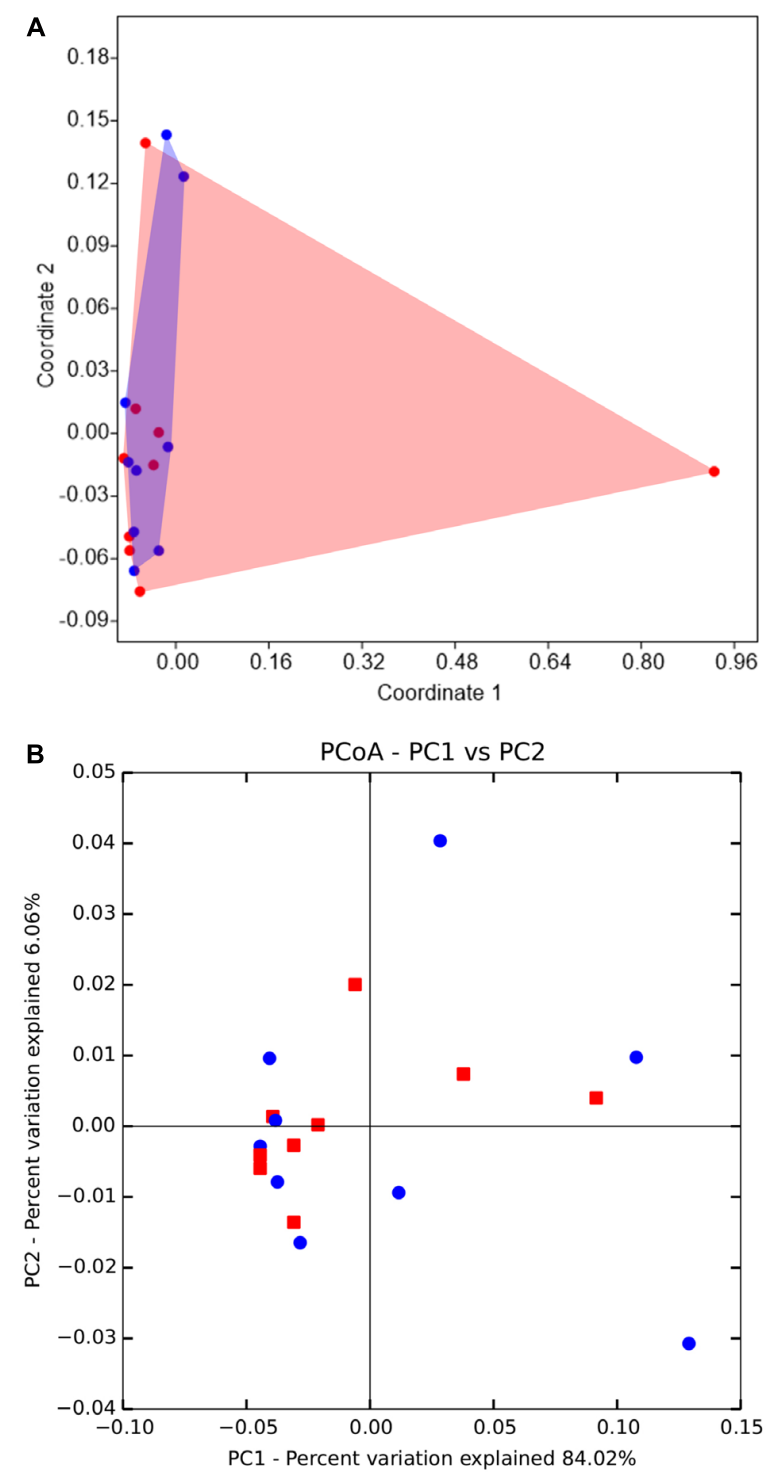

FIGURE 2 | Non-metric multidimensional scaling (NMDS) and principal coordinates analysis (PCOA) of the gut microbiota of Dendroctonus valens between low and high attack density groups. (A) NMDS diagrams of 18 samples, based on Bray-Curtis distance matrix for bacterial communities that consisted of OTUs ( $97 \%$ similarity level). Bacterial communities of the two groups were not separated from each other. (B) PCoA plots based on the weighted UniFrac metric for bacterial communities. Permutational multivariate analysis of variance indicated that there were no differences between bacterial communities of the two groups. The red represents samples from high density group, and blue represents samples from low density group.

abundance of genera Erwinia and Halomonas were the highest among all genera.

\section{Quantification of the Monoterpenes and Carbohydrates Concentrations}

Quantification of the monoterpenes in the phloem tissues results showed that $\alpha$-pinene, $\beta$-pinene, limonene concentration in the phloem of trees at high attack density are $1.48 \pm 0.13 \mathrm{mg} / \mathrm{g}$, $0.19 \pm 0.02 \mathrm{mg} / \mathrm{g}$, and $0.08 \pm 0.01 \mathrm{mg} / \mathrm{g}$ respectively, which are significantly higher than the concentration of the monoterpenes at the low attack density group (Table 2).

Quantification of the carbohydrates concentration (D-glucose, D-pinitol, and D-fructose) in the phloem tissues results showed that D-glucose, D-pinitol, and D-fructose concentration in the phloem at the high density group were not significantly different from the concentrations at the low attack density group (Figure 3), and D-glucose is the highest abundant carbohydrate in the phloem, D-pinitol is the lowest abundant carbohydrate.

\section{DISCUSSION}

Our results confirmed that the different attack densities of $D$. valens can influence host defensive monoterpenes concentration. $\alpha$-Pinene and $\beta$-pinene are the most abundant defensive monoterpenes for host $P$. tabulaeformis (Chen et al., 2006; Xu et al., 2014), and their concentrations rapidly accumulate after beetle attack (Miller et al., 1986; Leufvén and Birgersson, 1987). In comparison with the concentration of the monoterpenes in healthy tree ( $\alpha$-pinene, $0.52 \pm 0.17 \mathrm{mg} / \mathrm{g}$; $\beta$-pinene, $0.07 \pm 0.02 \mathrm{mg} / \mathrm{g}$; limonene, $0.02 \pm 0.01 \mathrm{mg} / \mathrm{g}$ ) (Xu et al., 2014), our results showed that monoterpenes concentrations are generally elevated by beetles' attack in both low and high attack density groups (Table 2). Furthermore, we found that its concentration in the phloem at high attack density is significantly higher than those at the low attack density (Table 2). Plant defenses are basically regulated by genetic factors, and many abiotic factors (e.g., light, ultraviolet radiation, seasonal variation, altitudinal variation, drought) and biotic factors (e.g., phytophagous insect, pathogens, fungus) have also been shown to influence its regulation (Close and McArthur, 2002; Solar et al., 2006; Spitaler et al., 2006; Adams et al., 2008; Ballare, 2014; Wang et al., 2015). Once being attacked by insects, plants release a variety of volatiles from the insect feeding damage site, and the profile of volatiles emitted is markedly different from those undamaged plants (Paré and Tumlinson, 1999; Forkner et al., 2004). Several studies showed that defensive monoterpenes of pine trees significantly increased after attack by beetles (Miller et al., 1986; Leufvén and Birgersson, 1987; Wallin and Raffa, 1999). It was also reported that some Chinese fungal associates of $D$. valens induced higher concentration of other defensive chemicals including diterpene resin acids and naringenin in P. tabuliformis (Cheng et al., 2015, 2016). In the study, whether or not the associated microorganisms of $D$. valens led to the variation of monoterpenes concentration needs further study.

Although monoterpene intensity was significantly different between the low attack density group and the high attack density group, no significant differences of $D$. valens gut bacterial community structure were found (Figure 2). This may partly be explained by the quick adaptation of gut microbiota to high concentration of $\alpha$-pinene (Xu et al., 2016c). It was reported that $\alpha$-pinene can alter $D$. valens gut bacterial community structure in $6 \mathrm{~h}$, but this change was recovered to the original bacterial community after $48 \mathrm{~h}$ (Xu et al., 2016c). Besides, diet has 
TABLE 2 | Monoterpene quantity (mg/g) (Mean \pm SEM) in Pinus tabuliformis phloem at low and high attack densities.

\begin{tabular}{|c|c|c|c|c|c|c|c|c|}
\hline \multirow[t]{2}{*}{ Chemical } & \multicolumn{3}{|c|}{ Low attack density } & \multicolumn{3}{|c|}{ High attack density } & \multirow[b]{2}{*}{$t$} & \multirow[b]{2}{*}{$P$} \\
\hline & Concentration & Minimum & Maximum & Concentration & Minimum & Maximum & & \\
\hline$\alpha$-Pinene & $0.44 \pm 0.04$ & 0.11 & 0.63 & $1.48 \pm 0.13$ & 1.08 & 2.47 & 7.65 & $<0.01$ \\
\hline$\beta$-Pinene & $0.07 \pm 0.01$ & 0.04 & 0.16 & $0.19 \pm 0.02$ & 0.12 & 0.35 & 4.83 & $<0.01$ \\
\hline Limonene & $0.04 \pm 0.01$ & 0.02 & 0.08 & $0.08 \pm 0.01$ & 0.04 & 0.12 & 5.12 & $<0.01$ \\
\hline
\end{tabular}

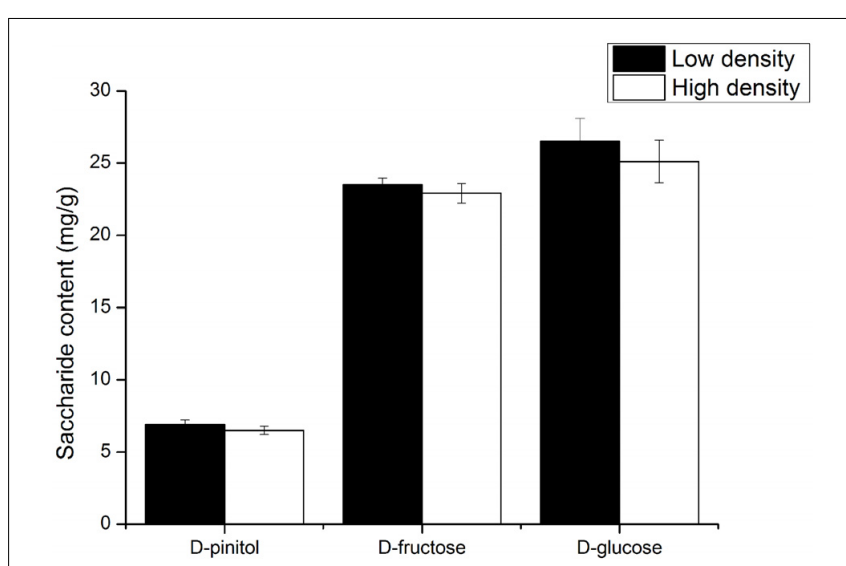

FIGURE 3 | The carbohydrates concentrations (D-glucose, D-pinitol, and D-fructose) in the pine phloem tissue at low and high attack density groups. The data were analyzed using independent $t$-test.

proved to play a major role in shaping gut bacterial communities for model insect Drosophila melanogaster and insects that ingest lignocellulose-derived substances (Chandler et al., 2011; Colman et al., 2012). But the carbohydrates concentration (D-glucose, D-pinitol, and D-fructose) in the diet of $D$. valens between the two groups were similar (Figure 3), which is independent of different attack density. Thus, whether different carbohydrates in diet would influence the gut microbiota need further study.

The most abundant genera in this study were Erwinia and Halomonas (Supplementary Table S2), which is not consistent with the results of gut microbiota in emerged insects which were captured in newly attacked pine stumps or fed phloem media without monoterpenes (Xu et al., 2015, 2016b). Erwinia are common gut bacteria in $D$. valens described in previous studies using both culture and uncultured method ( $\mathrm{Xu}$ et al., 2015, 2016b), but Halomonas was first reported in D. valens guts. Halomonas have been found in other insects system, e.g., the pine wilt disease insect vectors Monochamus galloprovincialis and M. alternatus (Alves et al., 2016) and pine weevil (Ölander, 2013). As the environmental acquisition of diverse microbes has been shown to lead to the change of gut bacterial assemblage (Mason and Raffa, 2014), D. valens may acquire the Halomonas bacteria from host environment, which needs further study to confirm e.g., setting a negative control to explore initial conditions of microbiota of emerged beetles. In addition, Halomonas are able to produce cellulase and have cellulolytic activity (Huang et al., 2010; Shivanand et al., 2013). The genome analysis of Halomonas sp. strain KO116 indicated that several relevant genes required for lignin degradation were highly observed in KO116 genome (Kameshwar and Qin, 2016). The phloem of P. tabuliformis is rich in cellulose, thus, the high abundance of Halomonas in the gut of $D$. valens may facilitate its nutrients uptake. Besides, $D$. valens in host pines trees at both low and high attack densities have a relative stable gut bacterial community structure (Figure 2), which is also important for the communities to conduct ecological functions.

Our result showed D-glucose concentration in the phloem is the highest and D-pinitol is the lowest abundant carbohydrate (Figure 3), which is not consistent with the results executed in healthy $P$. tabuliformis (D-pinitol, $21.96 \pm 4.10 \mathrm{mg} / \mathrm{g}$; D-fructose, $14.82 \pm 3.68 \mathrm{mg} / \mathrm{g}$; D-glucose, $18.12 \pm 6.65 \mathrm{mg} / \mathrm{g}$ ) (Zhou et al., 2016). The phenomenon may attribute to the bacteria-fungi interactions associated with $D$. valens that regulate carbohydrate concentration in the phloem. Several dominant culturable bacteria including Pseudomonas associated with D. valens inhibited D-glucose consumption of Ophiostoma minus and forced Leptographium procerum to consume D-pinitol prior to D-glucose, thus, may lead to increase in D-glucose concentration and decrease in D-pinitol concentration (Zhou et al., 2016). Although soluble sugars and starch content in phloem were significantly changed after $72 \mathrm{~h}$ attack initiated by bark beetles (Miller et al., 1986), previous studies also suggested that carbohydrates contents were changed by beetles' attack after several weeks or months (Dunn and Lorio, 1992; Wiley et al., 2016), thus $72 \mathrm{~h}$ after the attack of $D$. valens may not long enough to induce the variation of carbohydrates content in pine trees. In future experiments, we plan to prolong the sampling time after the attack of $D$. valens and inoculate the bacteria and fungi to the phloem directly to confirm whether the interactions contribute to the result.

\section{AUTHOR CONTRIBUTIONS}

The study was jointly conceived by DX, LX, FZ, BW, SW, ML, and JS. Experiments were designed by DX, LX, FZ, SW, and ML. DX, LX, FZ, and BW prepared the manuscript. LX, DX, FZ, and ML edited the manuscript. DX, LX, and SW carried out the experiments.

\section{FUNDING}

We acknowledge the National Key Research and Development Program (2017YFD0600101), Frontier Science Key Project of the 
Chinese Academy of Sciences (QYZDJ-SSW-SMC024), National Natural Science Foundation of China (31700569), and the China Postdoctoral Science Foundation (2017M622389).

\section{ACKNOWLEDGMENTS}

We thank Dr. Jacob Wickham (Institute of Zoology, CAS and Managing editor for Integrative Zoology) for his reviews of earlier versions of the manuscript.

\section{REFERENCES}

Adams, J. M., Rehill, B., Zhang, Y., and Gower, J. (2008). A test of the latitudinal defense hypothesis: herbivory, tannins and total phenolics in four North American tree species. Ecol. Res. 24, 697-704. doi: 10.1007/s11284-0080541-x

Alves, M., Pereira, A., Matos, P., Henriques, J., Vicente, C., Aikawa, T., et al. (2016). Bacterial community associated to the pine wilt disease insect vectors Monochamus galloprovincialis and Monochamus alternatus. Sci. Rep. 6:23908. doi: $10.1038 /$ srep 23908

Ballare, C. L. (2014). Light regulation of plant defense. Annu. Rev. Plant Biol. 65, 335-363. doi: 10.1146/annurev-arplant-050213-040145

Birgersson, G., Schlyter, F., Löfqvist, J., and Bergström, G. (1984). Quantitative variation of pheromone components in the spruce bark beetle Ips typographus from different attack phases. J. Chem. Ecol. 10, 1029-1055. doi: 10.1007/ BF00987511

Blumberg, B. J., Trop, S., Das, S., and Dimopoulos, G. (2013). Bacteria-and IMD pathway-independent immune defenses against Plasmodium falciparum in Anopheles gambiae. PLoS One 8:e72130. doi: 10.1371/journal.pone.007 2130

Boone, C. K., Keefover-Ring, K., Mapes, A. C., Adams, A. S., Bohlmann, J., and Raffa, K. F. (2013). Bacteria associated with a tree-killing insect reduce concentrations of plant defense compounds. J. Chem. Ecol. 39, 1003-1006. doi: 10.1007/s10886-013-0313-0

Briones-Roblero, C. I., Hernández-García, J. A., Gonzalez-Escobedo, R., SotoRobles, L. V., Rivera-Orduña, F. N., and Zúñiga, G. (2017). Structure and dynamics of the gut bacterial microbiota of the bark beetle, Dendroctonus rhizophagus (Curculionidae: Scolytinae) across their life stages. PLoS One 12:e0175470. doi: 10.1371/journal.pone.0175470

Byers, J. (1981). Pheromone biosynthesis in the bark beetle, Ips paraconfusus, during feeding or exposure to vapours of host plant precursors. Insect Biochem. 11, 563-569. doi: 10.1016/0020-1790(81)90024-X

Cao, Q., Wickham, J. D., Chen, L., Ahmad, F., Lu, M., and Sun, J. (2018). Effect of oxygen on verbenone conversion from cis-verbenol by gut facultative anaerobes of Dendroctonus valens. Front. Microbiol. 9:464. doi: 10.3389/fmicb.2018.00464

Caporaso, J. G., Kuczynski, J., Stombaugh, J., Bittinger, K., Bushman, F. D., Costello, E. K., et al. (2010). QIIME allows analysis of high-throughput community sequencing data. Nat. Methods 7, 335-336. doi: 10.1038/nmeth. f.303

Chandler, J. A., Morgan Lang, J., Bhatnagar, S., Eisen, J. A., and Kopp, A. (2011). Bacterial communities of diverse Drosophila species: ecological context of a host-microbe model system. PLoS Genet. 7:e1002272. doi: 10.1371/journal. pgen. 1002272

Chen, H., Tang, M., Gao, J., Chen, X., and Li, Z. (2006). Changes in the composition of volatile monoterpenes and sesquiterpenes of Pinus armandi, P. tabulaeformis, and P. bungeana in Northwest China. Chem. Nat. Comp. 42, 534-538. doi: 10.1007/s10600-006-0208-1

Cheng, C., Xu, L., Xu, D., Lou, Q., Lu, M., and Sun, J. (2016). Does cryptic microbiota mitigate pine resistance to an invasive beetle-fungus complex? Implications for invasion potential. Sci. Rep. 6:33110. doi: 10.1038/srep33110

Cheng, C., Zhou, F., Lu, M., and Sun, J. (2015). Inducible pine rosin defense mediates interactions between an invasive insect-fungal complex and newly acquired sympatric fungal associates. Integr. Zool. 10, 453-464. doi: 10.1111/ $1749-4877.12138$

\section{SUPPLEMENTARY MATERIAL}

The Supplementary Material for this article can be found online at: https://www.frontiersin.org/articles/10.3389/fmicb. 2018.01251/full\#supplementary-material

TABLE S1 | List of OTU shared between gut microbiota of bark beetles in low and high attack density groups.

TABLE S2 | Abundance of all genera in each sample. The abundance is presented in terms of percentages of the total sequences in a sample.

Close, D. C., and McArthur, C. (2002). Rethinking the role of many plant phenolics - protection from photodamage not herbivores? Oikos 99, 166-172. doi: 10.1034/j.1600-0706.2002.990117.x

Colman, D., Toolson, E., and Takacs-Vesbach, C. (2012). Do diet and taxonomy influence insect gut bacterial communities? Mol. Ecol. 21, 5124-5137. doi: 10.1111/j.1365-294X.2012.05752.x

Coulson, R. N. (1979). Population dynamics of bark beetles. Annu. Rev. Entomol. 24, 417-447. doi: 10.1146/annurev.en.24.010179.002221

DeSantis, T. Z., Hugenholtz, P., Larsen, N., Rojas, M., Brodie, E. L., Keller, K., et al. (2006). Greengenes, a chimera-checked 16S rRNA gene database and workbench compatible with ARB. Appl. Environ. Microbiol. 72, 5069-5072. doi: 10.1128/AEM.03006-05

Dillon, R., Vennard, C., and Charnley, A. (2002). A note: gut bacteria produce components of a locust cohesion pheromone. J. Appl. Microbiol. 92, 759-763. doi: 10.1046/j.1365-2672.2002.01581.x

Dillon, R. J., and Dillon, V. M. (2004). The gut bacteria of insects: nonpathogenic interactions. Annu. Rev. Entomol. 49, 71-92. doi: 10.1146/annurev.ento.49. 061802.123416

Dunn, J. P., and Lorio, P. L. (1992). Effects of bark girdling on carbohydrate supply and resistance of loblolly pine to southern pine beetle (Dendroctonus frontalis Zimm.) attack. For. Ecol. Manag. 50, 317-330. doi: 10.1016/0378-1127(92) 90345-A

Durand, A. A., Bergeron, A., Constant, P., Buffet, J. P., Deziel, E., and Guertin, C. (2015). Surveying the endomicrobiome and ectomicrobiome of bark beetles: the case of Dendroctonus simplex. Sci. Rep. 5:17190. doi: 10.1038/srep17190

Edgar, R. C. (2013). UPARSE: highly accurate OTU sequences from microbial amplicon reads. Nat. Methods 10, 996-998. doi: 10.1038/nmeth.2604

Edgar, R. C., Haas, B. J., Clemente, J. C., Quince, C., and Knight, R. (2011). UCHIME improves sensitivity and speed of chimera detection. Bioinformatics 27, 2194-2200. doi: 10.1093/bioinformatics/btr381

Engel, P., and Moran, N. A. (2013). The gut microbiota of insects - diversity in structure and function. FEMS Microbiol. Rev. 37, 699-735. doi: 10.1111/15746976.12025

Forkner, R. E., Marquis, R. J., and Lill, J. T. (2004). Feeny revisited: condensed tannins as anti-herbivore defences in leaf-chewing herbivore communities of Quercus. Ecol. Entomol. 29, 174-187. doi: 10.1111/j.1365-2311.2004.0590.x

Gao, B., Wen, X., Guan, H., Knizek, M., and Zdarek, J. (2005). Distribution and attack behavior of the red turpentine beetle, Dendroctonus valens, recently introduced to China. J. For. Sci. 51, 155-160. doi: 10.17221/4554-JFS

García-Fraile, P. (2018). Roles of bacteria in the bark beetle holobiont - how do they shape this forest pest? Ann. Appl. Biol. 172, 111-125. doi: 10.1111/aab. 12406

Genta, F. A., Dillon, R. J., Terra, W. R., and Ferreira, C. (2006). Potential role for gut microbiota in cell wall digestion and glucoside detoxification in Tenebrio molitor larvae. J. Insect Physiol. 52, 593-601. doi: 10.1016/j.jinsphys.2006.02.007

Gitau, C., Bashford, R., Carnegie, A., and Gurr, G. (2013). A review of semiochemicals associated with bark beetle (Coleoptera: Curculionidae: Scolytinae) pests of coniferous trees: a focus on beetle interactions with other pests and their associates. For. Ecol. Manag. 297, 1-14. doi: 10.1016/j.foreco. 2013.02.019

Guérard, N., Dreyer, E., and Lieutier, F. (2000). Interactions between Scots pine, Ips acuminatus (Gyll.) and Ophiostoma brunneo-ciliatum (Math.): estimation of the critical thresholds of attack and inoculation densities and effects on hydraulic properties in the stem. Ann. For. Sci. 57, 681-690. doi: 10.1051/forest:2000149 
Hamady, M., Lozupone, C., and Knight, R. (2010). Fast UniFrac: facilitating highthroughput phylogenetic analyses of microbial communities including analysis of pyrosequencing and PhyloChip data. ISME J. 4, 17-27. doi: 10.1038/ismej. 2009.97

Hammer, Ø., Harper, D., and Ryan, P. (2001). PAST: paleontological statistics software package for education and data analysis. Palaeontol. Electron 4, 1-9. doi: 10.1016/j.forsciint.2011.11.010

Hedden, R., and Pitman, G. (1978). Attack density regulation: a new approach to the use of pheromones in Douglas-fir beetle population management. J. Econ Entomol. 71, 633-637. doi: 10.1093/jee/71.4.633

Hernández-García, J. A., Briones-Roblero, C. I., Rivera-Orduña, F. N., and Zúñiga, G. (2017). Revealing the gut bacteriome of Dendroctonus bark beetles (Curculionidae: Scolytinae): diversity, core members and co-evolutionary patterns. Sci. Rep. 7:13864. doi: 10.1038/s41598-017-14031-6

Howe, M., Keefover-Ring, K., Raffa, K. F., and Louis, J. (2018). Pine engravers carry bacterial communities whose members reduce concentrations of host monoterpenes with variable degrees of redundancy, specificity, and capability. Environ. Entomol. doi: 10.1093/ee/nvy032 [Epub ahead of print].

Huang, X., Shao, Z., Hong, Y., Lin, L., Li, C., Huang, F., et al. (2010). Cel8H, a novel endoglucanase from the halophilic bacterium Halomonas sp. S66-4: molecular cloning, heterogonous expression, and biochemical characterization. J. Microbiol. 48, 318-324. doi: 10.1007/s12275-0090188-5

Kameshwar, A. K., and Qin, W. (2016). Recent developments in using advanced sequencing technologies for the genomic studies of lignin and cellulose degrading microorganisms. Int. J. Biol. Sci. 12, 156-171. doi: 10.7150/ijbs. 13537

Kikuchi, Y., Hayatsu, M., Hosokawa, T., Nagayama, A., Tago, K., and Fukatsu, T. (2012). Symbiont-mediated insecticide resistance. Proc. Natl. Acad. Sci. U.S.A. 109, 8618-8622. doi: 10.1073/pnas.1200231109

Koch, H., and Schmid-Hempel, P. (2011). Socially transmitted gut microbiota protect bumble bees against an intestinal parasite. Proc. Natl. Acad. Sci. U.S.A. 108, 19288-19292. doi: 10.1073/pnas.1110474108

Leufvén, A., and Birgersson, G. (1987). Quantitative variation of different monoterpenes around galleries of Ips typographus (Coleoptera: Scolytidae) attacking Norway spruce. Can. J. Bot. 65, 1038-1044. doi: 10.1139/b87-144

Lisec, J., Schauer, N., Kopka, J., Willmitzer, L., and Fernie, A. R. (2006). Gas chromatography mass spectrometry-based metabolite profiling in plants. Nat. Protoc. 1, 387-396. doi: 10.1038/nprot.2006.59

Lyon, R. (1958). A useful secondary sex character in Dendroctonus bark beetles. Can. Entomol. 90, 582-584. doi: 10.4039/Ent90582-10

Mason, C., Rubert-Nason, K., Lindroth, R., and Raffa, K. (2015). Aspen defense chemicals influence midgut bacterial community composition of gypsy moth. J. Chem. Ecol. 41, 75-84. doi: 10.1007/s10886-014-0530-1

Mason, C. J., and Raffa, K. F. (2014). Acquisition and structuring of midgut bacterial communities in gypsy moth (Lepidoptera: Erebidae) larvae. Environ. Entomol. 43, 595-604. doi: 10.1603/EN14031

Miller, R. H., Berryman, A. A., and Ryant, C. A. (1986). Biotic elicitors of defense reactions in lodgepole pine. Phytochemistry 25, 611-612. doi: 10.1016/00319422(86)88008-6

Morales-Jiménez, J., De León, A. V.-P., García-Domínguez, A., MartínezRomero, E., Zúñiga, G., and Hernández-Rodríguez, C. (2013). Nitrogen-fixing and uricolytic bacteria associated with the gut of Dendroctonus rhizophagus and Dendroctonus valens (Curculionidae: Scolytinae). Microb. Ecol. 66, 200-210. doi: 10.1007/s00248-013-0206-3

Muhammad, A., Fang, Y., Hou, Y., and Shi, Z. (2017). The gut entomotype of red palm weevil Rhynchophorus ferrugineus Olivier (Coleoptera: Dryophthoridae) and their effect on host nutrition metabolism. Front. Microbiol. 8:2291. doi: $10.3389 /$ fmicb.2017.02291

Ölander, T. (2013). Culture Independent Analysis of Microbiota in the Gut of Pine Weevils. Master's thesis, KTH Royal Institute of Technology, Stockholm.

Paine, T., Raffa, K., and Harrington, T. (1997). Interactions among scolytid bark beetles, their associated fungi, and live host conifers. Annu. Rev. Entomol. 42, 179-206. doi: 10.1146/annurev.ento.42.1.179

Paré, P. W., and Tumlinson, J. H. (1999). Plant volatiles as a defense against insect herbivores. Plant Physiol. 121, 325-332. doi: 10.1104/pp.121. 2.325
Phillips, M. A., and Croteau, R. B. (1999). Resin-based defenses in conifers. Trends Plant Sci. 4, 184-190. doi: 10.1016/S1360-1385(99)01401-6

Raffa, K., and Berryman, A. (1983). The role of host plant resistance in the colonization behavior and ecology of bark beetles (Coleoptera: Scolytidae). Ecol. Monogr. 53, 27-49. doi: 10.2307/1942586

Rodrigues, J., Brayner, F. A., Alves, L. C., Dixit, R., and Barillas-Mury, C. (2010). Hemocyte differentiation mediates innate immune memory in Anopheles gambiae mosquitoes. Science 329, 1353-1355. doi: 10.1126/science. 1190689

Seybold, S. J., Huber, D. P., Lee, J. C., Graves, A. D., and Bohlmann, J. (2006). Pine monoterpenes and pine bark beetles: a marriage of convenience for defense and chemical communication. Phytochem. Rev. 5, 143-178. doi: 10.1007/s11101006-9002-8

Sharon, G., Segal, D., Ringo, J. M., Hefetz, A., Zilber-Rosenberg, I., and Rosenberg, E. (2010). Commensal bacteria play a role in mating preference of Drosophila melanogaster. Proc. Natl. Acad. Sci. U.S.A. 107, 20051-20056. doi: 10.1073/pnas.1009906107

Shivanand, P., Mugeraya, G., and Kumar, A. (2013). Utilization of renewable agricultural residues for the production of extracellular halostable cellulase from newly isolated Halomonas sp. strain PS47. Ann. Microbiol. 63, 1257-1263. doi: 10.1007/s13213-012-0583-8

Smith, R. H. (1963). Toxicity of pine resin vapors to three species of Dendroctonus bark beetles. J. Econ. Entomol. 56, 827-831. doi: 10.1093/jee/56.6.827

Solar, A., Colarič, M., Usenik, V., and Stampar, F. (2006). Seasonal variations of selected flavonoids, phenolic acids and quinones in annual shoots of common walnut (Juglans regia L.). Plant Sci. 170, 453-461. doi: 10.1016/j.plantsci.2005. 09.012

Spitaler, R., Schlorhaufer, P. D., Ellmerer, E. P., Merfort, I., Bortenschlager, S., Stuppner, H., et al. (2006). Altitudinal variation of secondary metabolite profiles in flowering heads of Arnica montana cv. ARBO. Phytochemistry 67, 409-417. doi: 10.1016/j.phytochem.2005.11.018

Sullivan, B. T. (2016). "Chapter four - semiochemicals in the natural history of southern pine beetle Dendroctonus frontalis Zimmermann and their role in pest management," in Advances in Insect Physiology, eds C. Tittiger and G. J. Blomquist (Cambridge, MA: Academic Press), 129-193.

Sun, J., Lu, M., Gillette, N. E., and Wingfield, M. J. (2013). Red turpentine beetle: innocuous native becomes invasive tree killer in China. Annu. Rev. Entomol. 58, 293-311. doi: 10.1146/annurev-ento-120811-153624

Vasanthakumar, A., Delalibera, I., Handelsman, J., Klepzig, K. D., Schloss, P. D., and Raffa, K. F. (2006). Characterization of gut-associated bacteria in larvae and adults of the southern pine beetle, Dendroctonus frontalis Zimmermann. Environ. Entomol. 35, 1710-1717. doi: 10.1093/ee/35. 6.1710

Visotto, L. E., Oliveira, M. G., Guedes, R. N., Ribon, A. O., and Good-God, P. I. (2009). Contribution of gut bacteria to digestion and development of the velvetbean caterpillar, Anticarsia gemmatalis. J. Insect Physiol. 55, 185-191. doi: 10.1016/j.jinsphys.2008.10.017

Wallin, K. F., and Raffa, K. F. (1999). Altered constitutive and inducible phloem monoterpenes following natural defoliation of Jack pine: implications to host mediated interguild interactions and plant defense theories. J. Chem. Ecol. 25, 861-880. doi: 10.1023/A:1020853019309

Wang, Q., Garrity, G. M., Tiedje, J. M., and Cole, J. R. (2007). Naive Bayesian classifier for rapid assignment of rRNA sequences into the new bacterial taxonomy. Appl. Environ. Microbiol. 73, 5261-5267. doi: 10.1128/AEM. 00062-07

Wang, X., Gao, W., Liu, Q., Ni, Y., and Jiang, Z. (2015). Plant defensive strategies and environment-driven mechanisms. Chin. J. Ecol. 32, 3542-3552.

Wiley, E., Rogers, B. J., Hodgkinson, R., and Landhausser, S. M. (2016). Nonstructural carbohydrate dynamics of lodgepole pine dying from mountain pine beetle attack. New Phytol. 209, 550-562. doi: 10.1111/nph. 13603

Wood, D. L. (1982). The role of pheromones, kairomones, and allomones in the host selection and colonization behavior of bark beetles. Annu. Rev. Entomol. 27, 411-446. doi: 10.1146/annurev.en.27.010182.002211

Xu, B. B., Liu, Z. D., and Sun, J. H. (2014). The effects of $\alpha$-pinene on the feeding performance and pheromone production of Dendroctonus valens. Entomol. Exp. Appl. 150, 269-278. doi: 10.1111/eea.12161 
Xu, L., Lou, Q., Cheng, C., Lu, M., and Sun, J. (2015). Gut-associated bacteria of Dendroctonus valens and their involvement in verbenone production. Microb. Ecol. 70, 1012-1023. doi: 10.1007/s00248-015-0625-4

$\mathrm{Xu}, \mathrm{L} ., \mathrm{Lu}, \mathrm{M}$., and Sun, J. (2016a). Invasive bark beetle-associated microbes degrade a host defensive monoterpene. Insect Sci. 23, 183-190. doi: 10.1111/ 1744-7917.12255

Xu, L., Lu, M., Xu, D., Chen, L., and Sun, J. (2016b). Sexual variation of bacterial microbiota of Dendroctonus valens guts and frass in relation to verbenone production. J. Insect Physiol. 95, 110-117. doi: 10.1016/j.jinsphys.2016. 09.014

Xu, L., Shi, Z., Wang, B., Lu, M., and Sun, J. (2016c). Pine defensive monoterpene $\alpha$-pinene influences the feeding behavior of Dendroctonus valens and its gut bacterial community structure. Int. J. Mol. Sci. 17:E1734.

Yan, Z., Sun, J., Don, O., and Zhang, Z. (2005). The red turpentine beetle, Dendroctonus valens LeConte (Scolytidae): an exotic invasive pest of pine in China. Biodivers. Conserv. 14, 1735-1760. doi: 10.1007/s10531-004-0697-9

Zhang, Q. H., Birgersson, G., Schlyter, F., and Chen, G. F. (2000). Pheromone components in the larch bark beetle, Ips cembrae, from China: quantitative variation among attack phases and individuals. J. Chem. Ecol. 26, 841-858. doi: 10.1023/A:1005447922939

Zhou, F., Lou, Q., Wang, B., Xu, L., Cheng, C., Lu, M., et al. (2016). Altered carbohydrates allocation by associated bacteria-fungi interactions in a bark beetle-microbe symbiosis. Sci. Rep. 6:20135. doi: 10.1038/srep 20135

Conflict of Interest Statement: The authors declare that the research was conducted in the absence of any commercial or financial relationships that could be construed as a potential conflict of interest.

Copyright (c) $2018 \mathrm{Xu}, \mathrm{Xu}$, Zhou, Wang, Wang, Lu and Sun. This is an open-access article distributed under the terms of the Creative Commons Attribution License (CC BY). The use, distribution or reproduction in other forums is permitted, provided the original author(s) and the copyright owner are credited and that the original publication in this journal is cited, in accordance with accepted academic practice. No use, distribution or reproduction is permitted which does not comply with these terms. 